\title{
CLOUD AND AEROSOL INTERACTION OBSERVED IN SKYNET HEFEI SITE IN CHINA
}

\author{
Yingjian Wang ${ }^{1,3}$, Lu Sun ${ }^{1,2}$, Dong Liu ${ }^{1}$, Zhien Wang ${ }^{1,4}$, Zhenzhu Wang ${ }^{1}$, Chenbo Xie ${ }^{1}$ \\ ${ }^{1}$ Key Laboratory of Atmospheric Composition and Optical Radiation, Anhui Institute of Optics and Fine \\ Mechanics, Chinese Academy of Sciences, Hefei, Anhui 230031, China \\ ${ }^{2}$ University of Chinese Academy of Sciences, Beijing 100049, China \\ ${ }^{3}$ University of Science and Technology of China, Hefei, Anhui 230026, China \\ ${ }^{4}$ Department of Atmospheric Sciences, University of Wyoming, WY 82070, USA \\ *Email:wyj@aiofm.ac.cn
}

\begin{abstract}
The interaction relationship between cloud and aerosol is studied via their optical depth and cloud effective radius based on ground-based remote sensors. By attenuated backscatter obtained by lidar the optical depth of cloud and aerosol is retrieved. Combing with liquid water path observed by microwave radiometer, the cloud number concentration and cloud effective radius is also retrieved based on the adiabatic hypothesis Cases studies shows that during the stable stratocumulus with interval precipitation period and aerosol with vertical motion, the cloud effective radius shows both negative and positive relationship with aerosol optical depth. It may be due to the difference of liquid water path of the cloud properties and shows complex interaction with aerosol.
\end{abstract}

\section{INTRODUCTION}

Cloud and aerosol interaction and their climate effect have been at cutting edge of global climate change research since nineteen nineties. The 5th assessment report of Intergovernmental Panel on Climate Change (IPCC 2013) still consider that aerosol-cloud-precipitation interaction and its climate effect is one of the most uncertain factors in climate changing study. As the physical process of aerosol-cloud-radiation-precipitation interaction is rater complex, there are still many unknown issues and misunderstandings which are needed to make clear. Therefore, most process and parametrization cannot be presented in the numerical model exactly. If we hope to improve the parameterization schemes, we need to learn the 3D structures of clouds and aerosol, their microphysical properties and their interaction. Because of lacking relevant observational data, most of the parameterization schemes used in our country are not well-developed. It has become barrier of the research of climate change.

Combined observation by lidar and microwave radiometer from 16th to 17th, November, 2012 and from 13th to 14th, December, 2012 are chosen to retrieve the cloud microphysical properties and study their interaction with local aerosol.

\section{INSTRUMENTS AND DATASET}

SKYNET Hefei site $\left(31.89^{\circ} \mathrm{N}, 117.17^{\circ} \mathrm{E}\right)$ is one of the super observation sites in SKYNET program. This super site consists of a NIES twowavelength depolarization lidar at the wavelengths of $532 \mathrm{~nm}$ and $1064 \mathrm{~nm}$ and depolarization at $532 \mathrm{~nm}$, a sky radiometer, a microwave radiometer, a sky camera and some other radiation instruments with data archived from October, 2002 till now. The photos and main specifications of NIES two-wavelength depolarization lidar and two-band microwave radiometer is shown in fig 1,2 and table 1, 2 .

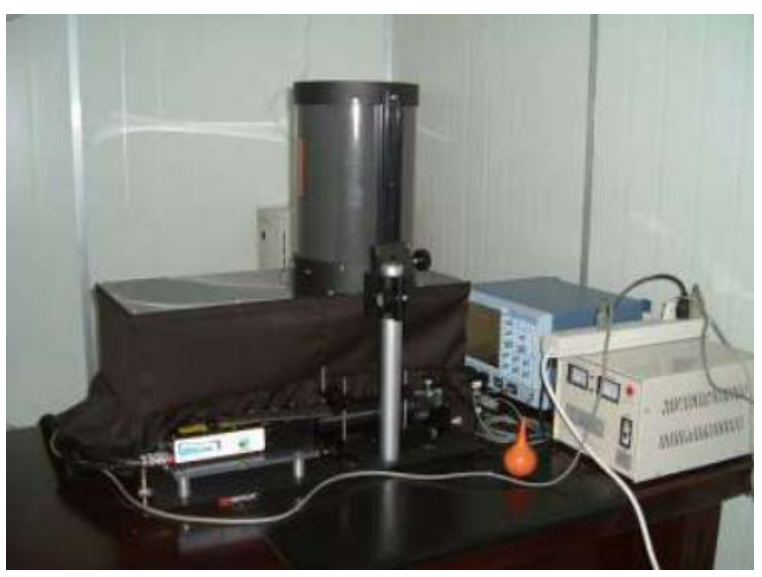

Fig. 1 Two-wavelength depolarization lidar initialized in SKYNET Hefei site by NIES, Japan 


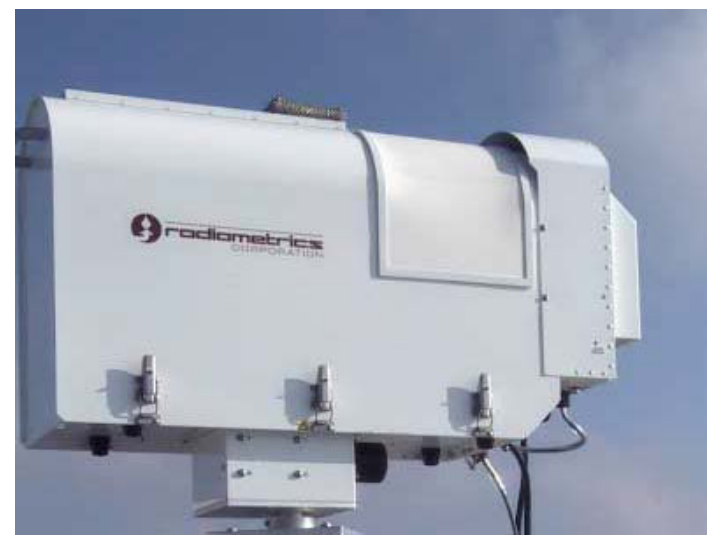

Fig.2 Two-band microwave radiometer installed in Hefei by SKYNET

Table1. The specifications of NIES two-wavelength depolarization lidar

\begin{tabular}{|l|c|}
\hline Laser & Nd:YAG(532,1064nm) \\
\hline Repetition & $10 \mathrm{~Hz}$ \\
\hline Power & $20 \mathrm{~mJ}$ \\
\hline Diameter of Telescope & $20 \mathrm{~cm}$ \\
\hline Direction & Zenith(fixed) \\
\hline Detector & PMT, APD \\
\hline Range Resolution & $7.5 \mathrm{~m}$ \\
\hline Signal Accumulation & 4000 shots (5min) \\
\hline Operation & 4 profiles/hour \\
\hline
\end{tabular}

Table2. The specification of two-band microwave radiometer

\begin{tabular}{|l|c|c|}
\hline Frequency/GHz & 23.8 & 31.4 \\
\hline Beam width ${ }^{\circ}$ & 5.8 & 4.5 \\
\hline Gain/dB & 31 & 33 \\
\hline Sampling Time & \multicolumn{2}{|c|}{$<1 \mathrm{~min}$} \\
\hline Resolution & \multicolumn{2}{|c|}{$0.25 \mathrm{~K}$} \\
\hline Accuracy & \multicolumn{2}{|c|}{$0.5 \mathrm{~K}$} \\
\hline $\begin{array}{l}\text { Angle Scanning } \\
\text { Range }\end{array}$ & $\begin{array}{c}\text { Azimuth } 3^{\circ} / \mathrm{s} ; \\
\text { Elevating angle }>90^{\circ} / \mathrm{s}\end{array}$ \\
\hline Scanning Speed
\end{tabular}

\section{ALGORITHMS AND RESULTS}

\subsection{Cloud vertical structure retrieval}

There exists a plenty of publications referred to the cloud vertical structure retrieval. We develop a universal algorithm which is suitable for most cases of high aerosol loading condition in China and our lidar system. Samples are shown that it works very well with our lidar system.
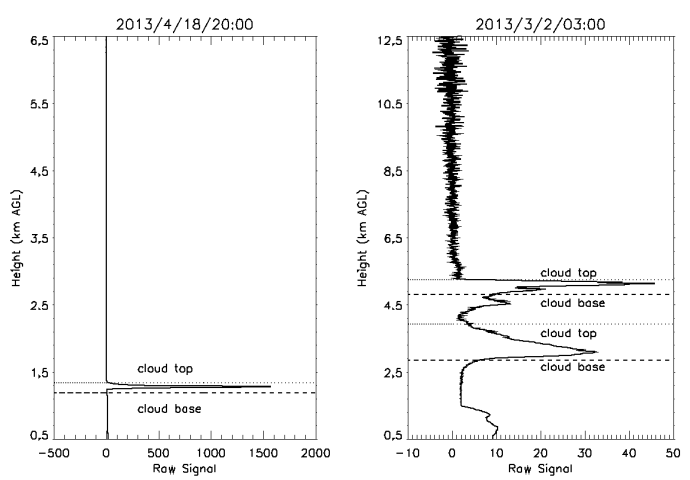

Fig.3. some cases of cloud vertical profile retrieval (left. low cloud ; right. multiple-layered cloud)

These are two vertical profiles that are typical cases including low cloud and multiple-layered cloud in Fig.3, one can see clearly that how it works faced with different kinds of cloud vertical profiles, single-layered and multiple-layered clouds. It shows that we can get exact cloud base and cloud top under different circumstance. Whereas, when it comes to multiple-layered cloud, it is hard for our lidar to detect because of the laser power and signal-noise-ratio. The right plot in Fig. 3 shows that the algorithm is suitable for detecting multiple-layered cloud.

\subsection{Cloud microphysical properties retrieval}

Based on the Robert Woods' field campaigns, the relationship between liquid water path and cloud optical depth as shown in the following is proposed:

$$
\begin{aligned}
& q_{L}(z)=f_{a d} \Gamma_{a d} z \\
& L W P=\frac{5}{9} \rho_{w} \tau r_{e}(h) \\
& L W P=\int_{z=0}^{h} q_{L} d z=\frac{1}{2} f_{a d} \Gamma_{a d} h^{2}
\end{aligned}
$$


$q_{L}$ is liquid water content $\left(\mathrm{g} / \mathrm{cm}^{2}\right), f_{a d}$ is the degree of adiabatic degree. It usually equals to 1 near cloud base. $\Gamma_{a d}$ is related to pressure and temperature. $r_{e}$ is cloud droplet effective radius. $\tau$ is cloud optical depth. Based on that and adiabatic assumption, cloud droplet effective radius could be calculated. Combining the aerosol optical depth observed by lidar and cloud droplet effective radius, we find in some typical cases, there exists a negative relationship between them. In the top panel Fig 4, it shows that the results match the Twomey effect (indirect effect of aerosol). However, in some cases, there exists opposite relationship between them as shown in the bottom panel in Fig 4. Comparing with others' observation results, it is possible because of the lack of liquid water path. If liquid water path is high enough for aerosol to become cloud condensation nuclei, there is negative relationship. If not, there is positive relationship because aerosol competes for liquid water to become cloud condensation nuclei.
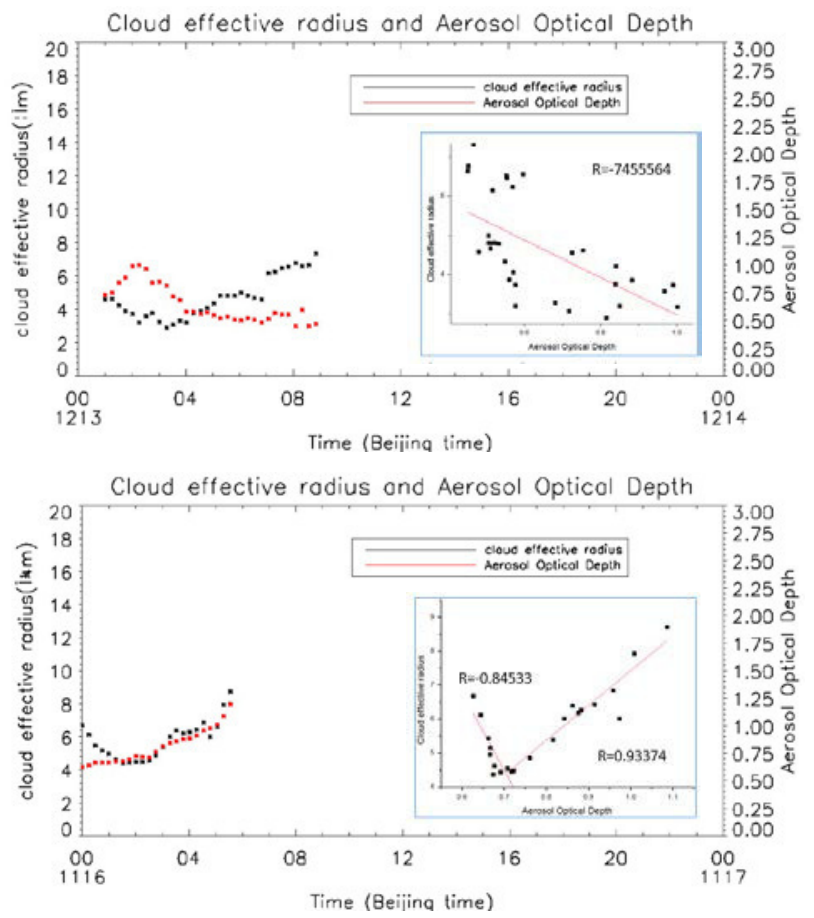

Fig 4 the relationship between cloud droplet effective radius and aerosol optical depth (top: negative relationship; bottom: positive relationship)

\section{CONCLUSION}

Due to the lack of observation instruments and temperature and humidity vertical profiles, it is difficult to derive exact cloud vertical structure. Moreover, aerosol retrieval method has been limited because there exists opaque thick cloud. If High Spectral Resolution Lidar has been adopted, aerosol extinction coefficient could be obtained without the assumption of lidar ratio. Based on multiple wavelength lidar, aerosol spectral distribution could be got. With Raman lidar, we could get water vapor and temperature vertical profiles. Combining lidar and Radar, cloud boundary could be acknowledged more exactly. So there is still possiblity for improvement in lidar-based cloud physical properties retrieval with state-of-art technology and long-term observation data.

Acknowledgement: This research has been supported by national basic research program of China (973 project) No. 2013CB955802, the project of Chinese Academy of Science No. XDA05100302, and NSCF No. 41075016. The authors would like to also thank Dr. Nobuo Sugimoto's group to initialize the lidar system in Hefei, China.

\section{References}

[1] Zhien Wang and Sassen, "Cloud Type and Macrophysical Property Retrieval Using Multiple Remote Sensors," Appl. Meteor, 40(10), 16651683(2001).

[2] Yongxiang Hu, Mark Vaughan, Zhaoyan Liu et al. Retrieving Optical Depths and Lidar Ratios for Transparent Layers Above Opaque Water Clouds From CALIPSO Lidar Measurements[J]. IEEE Geoscience and Remote Sensing Letters, 2007(4):523-526

[3] Jiming Li, Yongxiang Hu, Jianping Huang, et al. A New Method for Retrieval of the Extinction Coefficient of Water Clouds by Using the Tail of the CALIOP Signal [J]. Atmospheric Chemistry and Physics Discuss, 2010, 10: 28151-28181

[4] Robert Woods. Relationships between optical depth, liquid water path, droplet concentration and effective radius in an adiabatic layer cloud 